\section{Stimuli-responsive 2D polyelectrolyte photonic crystals for optically encoded $\mathrm{pH}$ sensing $\dagger$}

\author{
Cheng $\mathrm{Li}^{a b}$ and Bettina V. Lotsch*ab \\ Received 15th March 2012, Accepted 25th April 2012 \\ DOI: $10.1039 / \mathrm{c} 2 \mathrm{cc} 31916 \mathrm{k}$
}

A versatile photonic crystal sensing motif based on a twodimensional (2D) inverse opal monolayer of stimuli-responsive polyelectrolyte gel with tunable optical properties is reported. The photonic membrane shows prompt response to $\mathrm{pH}$ and can be readily read out from either its optical spectra or interference colours.

The development of sensing techniques is driven by the ever increasing demand for miniaturized sensing platforms with fast response in areas such as bioassays, environmental monitoring and disease diagnostics. ${ }^{1}$ Among the various kinds of sensing architectures, photonic crystal (PC) sensors that employ stimuliresponsive materials as signal transducers have been demonstrated to be promising in that they are able to perform sensitive detection in a non-destructive and label-free way. ${ }^{2}$ In past years, sensors have been developed by exploiting various PC motifs including opal films, ${ }^{3}$ inverse opals in the form of films ${ }^{4}$ or microparticles, ${ }^{5}$ Bragg stacks, ${ }^{6}$ porous silicon PCs, ${ }^{7}$ PC fibers, ${ }^{8}$ and microcavities. ${ }^{9}$ Among them, hydrogel photonic crystal sensors are of particular interest because they can be tailored to respond to various stimuli and show substantial volume changes upon recognition of analytes by swelling that could enhance the optical readout. ${ }^{3,4 a-g, 6 c, d}$ Asher's group developed hydrogel sensing materials by polymerizing $3 \mathrm{D}^{3 a-d}$ and most recently $2 \mathrm{D}$ colloidal crystal arrays, ${ }^{3 e}$ in which a volume change of the hydrogel upon recognition of the analyte led to modification of the optical thickness and therefore a shift in the position of the photonic stop band and, hence, a colour change. Based on a similar principle, 3D inverse opals of hydrogel have been shown to be applicable for the sensing of $\mathrm{pH},{ }^{4 a, e, g}$ glucose, ${ }^{4 b}$ temperature, ${ }^{4 c}$ and humidity. ${ }^{4 d}$ In terms of one dimensional PC architectures, Bragg stacks with hydrogel as a constituent layer have recently been demonstrated to be capable of humidity sensing. ${ }^{6 c, d}$ However, the above sensing motifs all suffer from slow response times when it comes to the sensing of solutes, such as $\mathrm{pH}$, or molecular analytes. It often takes more than tens of minutes or even hours to reach maximum response, which hampers applications in real-time sensing. The main reason for

${ }^{a}$ Max Planck Institute for Solid State Research, Heisenbergstrasse 1, 70569 Stuttgart, Germany

${ }^{b}$ Department of Chemistry and Center for Nanoscience,

Ludwig-Maximilians-Universität München, Butenandtstr. 5-13,

Haus D, 81377 München, Germany.E-mail:b.lotsch@fkf.mpg.de

$\dagger$ Electronic supplementary information (ESI) available. See DOI: $10.1039 / \mathrm{c} 2 \mathrm{cc} 31916 \mathrm{k}$ this is that the above sensing motifs are made from thick hydrogel structures (from several to tens of microns), which results in slow diffusion of solutes into the hydrogel and therefore slow response of the sensor. Although several measures have been taken to speed up the response, they lack feasibility and universality. ${ }^{3 e, 4 e}$

Herein, we report a versatile PC sensing motif, which is a stimuli-responsive 2D PC based on a monolayer inverse opal of polyelectrolyte gel (2DPC-PG). A remarkable feature of the 2DPC-PG is that it holds tunable photonic properties despite a sub-micron thickness. Therefore, the photonic sensing for solutes does not suffer from slow diffusion and the response can be greatly sped up.

To prepare 2DPC-PGs, a monolayer of a polystyrene (PS) colloidal array was first assembled on a glass or silicon substrate, onto which a solution of quaternized poly-(2-vinyl pyridine) (qP2VP) was spin-coated at the desired spin speed (ESI $\dagger)$. The PS monolayer was then selectively removed by dissolving it in toluene. Finally, a mechanically stable 2DPC-PG was obtained after thermal cross-linking at $120^{\circ} \mathrm{C}$. Fig. 1 shows the scanning electron microscopy (SEM) images of a 2DPC-PG prepared by using a monolayer opal assembled from $470 \mathrm{~nm}$ diameter PS spheres and a spin speed of $2000 \mathrm{rpm}$. From the SEM images, one can notice a thin layer covering the void arrays, which owing to its small thickness appears almost transparent to the electron beam in top view. It can further be seen in Fig. 1a that the P2VP gel replicates well the hexagonal order of the opal monolayer and the lattice parameter of the resulting 2DPC-PG corresponds to the diameter of the template PS spheres. The cross-section SEM image in Fig. 1b gives clear evidence of the top layer but at the same time shows that the height of the voids is decreased as compared to the original PS spheres,
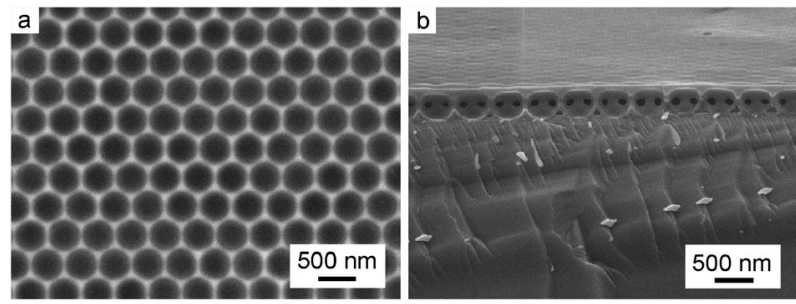

Fig. 1 SEM images of the 2D PC based on a monolayer inverse opal of polyelectrolyte gel (2DPC-PG) on a silicon wafer: (a) top-view and (b) side-view. 
suggesting a deformation of the voids from spherical to oblate, which can be attributed to the low stiffness of the gel. As a result, the overall thickness of the 2DPC-PG is less than the diameter of the PS spheres, which is further confirmed quantitatively by thickness measurement. The small holes in the walls of the voids are formed due to the close packing of the original PS spheres, indicating that the voids are interconnected with each other.

The structural parameters of the 2DPC-PG, i.e. its overall thickness and the parameters of the optical lattice can be readily tuned with the presented method. The mean thickness of the 2DPC-PG, which was measured with a profilometer, decreases almost linearly from 447, 417, 393, 363 to $332 \mathrm{~nm}$ as the spincoating speed gradually increases from 1000, 1500, 2000, 2500 to $3000 \mathrm{rpm}$ (Fig. S1, ESI $\dagger$ ). On the other hand, the lattice parameters of the 2DPC-PG can be conveniently varied by using a monolayer template with different sphere diameters. For example, 2DPC-PGs prepared with the same spin-coating speed of $2000 \mathrm{rpm}$ but sphere diameters of 470 and $535 \mathrm{~nm}$ possess lattice parameters of about 450 and $500 \mathrm{~nm}$ respectively (Fig. S2, ESI $\dagger$ ). However, they still have a similar mean thickness of around $395 \mathrm{~nm}$ as measured by profilometry. These results suggest that the thickness and the lattice parameter can be tuned separately, which enables us to study experimentally the relationship between structural parameters and optical properties of the 2DPC-PG.

The 2DPC-PG demonstrates tunable optical properties associated with its structural parameters. As shown in Fig. 2, while an unstructured/dense P2VP gel film prepared on a bare glass substrate is transparent (a), the 2DPC-PG samples (b-e) all show a major, well-defined dip in their transmission spectrum. Such a transmission dip corresponds to an attenuation of the transmitted intensity as the incoming waves couple to the photonic modes ${ }^{10}$ provided by the periodic structure of the 2DPC-PG (Scheme S1, ESI $\dagger$ ). The position of the transmission dip can be assigned to the lattice parameter of the periodic structure and described by $\lambda_{\mathrm{d}}=\sqrt{3} d n_{\mathrm{eff}} / 2,{ }^{10 d, e}$ where $d$ is the lattice parameter and $n_{\mathrm{eff}}$ is the effective refractive index (ESI $\dagger$ ). The dip position of 2DPC-PG is experimentally shown to be both thickness- and lattice parameter-dependent. For samples b, c and d prepared with $470 \mathrm{~nm}$ spheres, the transmission dip red-shifts from 471, via 485 to $502 \mathrm{~nm}$ with a decrease in spin-coating speed from 2500 , via 2000 to $1500 \mathrm{rpm}$ (i.e. an increase in overall thickness) as a result of an increasing $n_{\text {eff }}$. With a fixed spin-coating speed

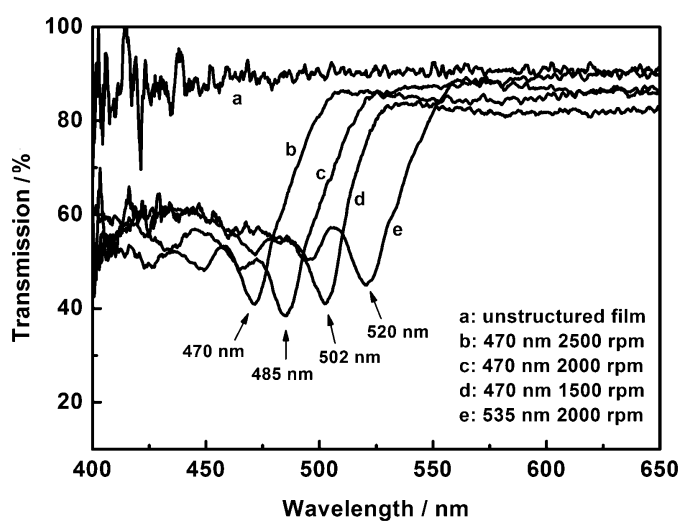

Fig. 2 Transmission spectra of an unstructured P2VP gel film and 2DPC-PGs prepared with varying sphere template diameters and spincoating speeds on glass substrates. a)

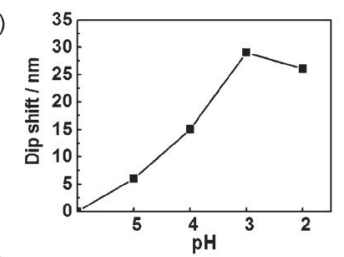

c)
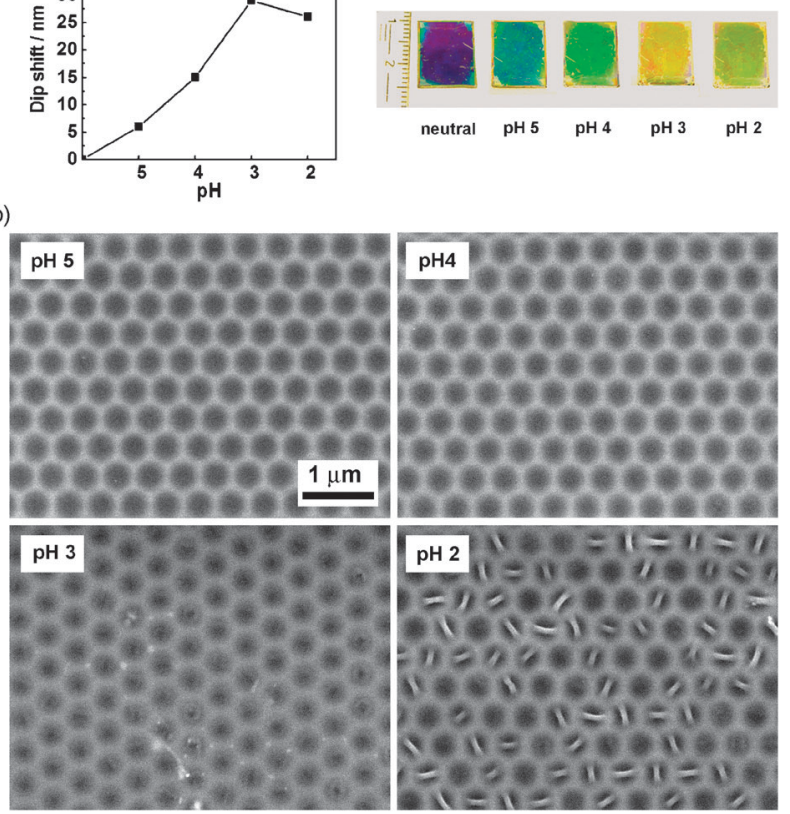

Fig. 3 Transmission dip shift (a), morphological changes (b) and interference colours (c) of 2DPC-PGs in response to different $\mathrm{pH}$ conditions. The 2DPC-PGs were prepared with $470 \mathrm{~nm}$ opal monolayers and a spin-coating speed of $2000 \mathrm{rpm}$ on glass (a) and silicon wafer $(b, c)$, respectively.

of $2000 \mathrm{rpm}$ and hence a constant thickness, the transmission dip of the samples c and e red-shifts from 485 to $520 \mathrm{~nm}$ as the template sphere diameter increases from 470 to $535 \mathrm{~nm}$.

The pronounced dependence of the $\mathrm{PC}$ feature (i.e. transmission dip) of the 2DPC-PG on its structural parameters as shown above makes it promising for optically encoded sensing, as the swelling of the polyelectrolyte gel upon external stimuli will lead to changes in the structural parameters that can be read out directly from the shift of the transmission dip. As P2VP is well known as a weak cationic polyelectrolyte that exhibits fast and substantial swelling under acidic conditions due to protonation of the pyridine group, ${ }^{11}$ a $\mathrm{pH}$ sensor based on 2DPC-PG is demonstrated in this report. Fig. 3a shows the transmission dip shift of a 2DPC-PG in response to $\mathrm{pH} 5,4,3$ and 2, which is 6, 15, 29 and $26 \mathrm{~nm}$ as compared to its initial position, respectively. Therefore, the variation of $\mathrm{pH}$ conditions can be readily read out from the wavelength shift of the 2DPC-PG's transmission dip. Besides, the swollen 2DPC-PGs can be entirely recovered by a quick wash with a basic solution or pure water. Accordingly, the optically encoded response of the 2DPC-PG upon $\mathrm{pH}$ changes is totally reversible and can be cycled more than 10 times without degradation (Fig. S3, ESI $\dagger$ ).

To explain the optical response, i.e. the transmission dip shift of the 2DPC-PG as a function of $\mathrm{pH}$, its related structural and morphological changes under different $\mathrm{pH}$ conditions was studied. Under acidic conditions the 2DPC-PG swells dramatically and its thickness increases by $5 \%, 21 \%, 37 \%$ and $34 \%$ after soaking in $\mathrm{pH} 5, \mathrm{pH} 4, \mathrm{pH} 3$ and $\mathrm{pH} 2$ solutions, respectively (Fig. S4 and S5, ESI $\dagger$ ). Such a trend in thickness variation is in complete agreement with the transmission dip shifts as shown above and indicates once more that the PC properties of 


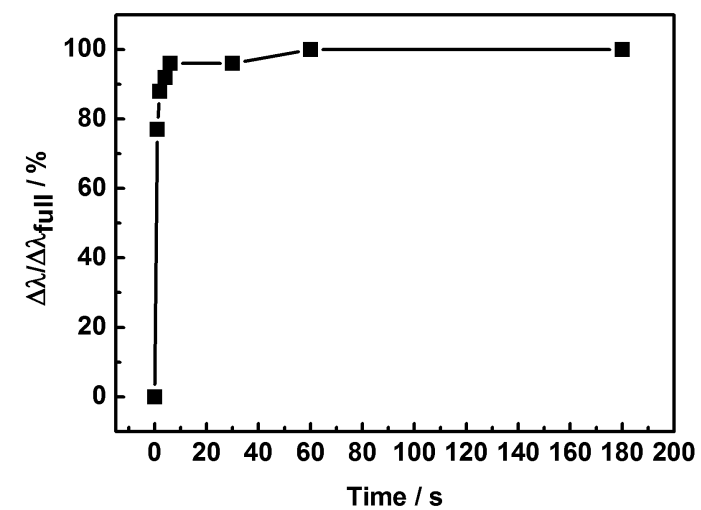

Fig. 4 Response kinetics of the 2DPC-PG pH sensor to $\mathrm{pH} 3$.

the 2DPC-PG are a faithful replication of its structural parameters. Moreover, the top-view SEM images of the swollen 2DPC-PGs under different $\mathrm{pH}$ conditions (Fig. 3b) reveals that the lattice parameter of the 2DPC-PG stays almost the same, although the inner voids gradually diminish from $\mathrm{pH} 5$ via $\mathrm{pH} 4$ to $\mathrm{pH} 3$. Notably, upon $\mathrm{pH} 2$ the suspended part of the overlayer in the 2DPC-PG exhibits significant wrinkling. The observed blue-shift of the transmission dip from $\mathrm{pH} 3$ to $\mathrm{pH} 2$ clearly correlates with a buckling and, hence, a decrease in the optical thickness of the membrane. The 2DPC-PG is anchored to a stiff substrate and is thus subject to a graded constraint in its swelling, but unlike a dense P2VP gel film which folds upon $\mathrm{pH} 2$ due to the constraint imposed by the substrate, ${ }^{11 b}$ its smaller contact area to the substrate endows it with higher flexibility and buckling deformation is restricted to the suspended top layer, thus avoiding a large range folding of the film. The structural stability upon exposure to external stimuli enables the 2DPC-PG to perform as a durable, reversible and mechanically strong $\mathrm{pH}$ sensor.

Apart from the transmission dip arising from the in-plane waveguide-like mode and hence, the inherent $\mathrm{PC}$ properties of the membrane, the 2DPC-PG exhibits vivid colour when prepared on highly reflecting substrates as a result of optical interference, which can also be exploited as a visibly perceptible indicator for sensing events. Fig. 3c shows in a row the photographs of a 2DPC-PG that was prepared on a silicon substrate before and after soaking in solutions of $\mathrm{pH} \mathrm{5,4,3}$ and 2 and subsequent drying. The varying hues from purple, blue, green to yellow upon different $\mathrm{pH}$ conditions are noticeable to the naked eye. The Fabry-Pérot interference fringes of the 2DPC-PGs within the visible region exhibit shifts in complete agreement with the observed optical and thickness changes (Fig. S6, ESI $\dagger$ ).

Remarkably, the 2DPC-PG pH sensor exhibits a prompt optical response. As shown in Fig. 4, within 6 seconds soaking in a solution of $\mathrm{pH} 3$ the transmission dip shift already reaches $96 \%$ of the full response after 1 minute, indicating that equilibrium can be well established within seconds. Recently, Asher's group reported a powerful 2D PC hydrogel $\mathrm{pH}$ sensor that however needs 30 minutes to reach its full response. ${ }^{3 e}$ Compared with the PC $\mathrm{pH}$ sensors designed so far, the 2DPC-PG presents the thinnest gel structure, resulting in the observed fastest response times.
In summary, we demonstrate the successful fabrication of 2D inverse opal monolayers out of a swellable P2VP polyelectrolyte gel by a facile spin-coating method. The stimuliresponsive membranes give rise to a versatile photonic crystal $\mathrm{pH}$ sensing motif based on their tunable optical properties and exhibit fast response times owing to their sub-micron thickness. Such a 2D inverse opal sensing motif can be easily extended for various sensing events when coupled with tailormade responsive materials.

Dr C. Li acknowledges support from the Alexander von Humbolt Foundation. The authors thank Dr I. Tokarev and Prof. V. V. Tsukruk for useful discussions and Dr Steffen Schmid and Viola Duppel for SEM measurements. Financial support from the Nanosystems Initiative Munich (NIM), Center for Nanoscience (CeNS) and Fonds der Chemischen Industrie (FCI) is gratefully acknowledged.

\section{Notes and references}

1 (a) M. D. Porter, R. J. Lipert, L. M. Siperko, G. Wang and R. Narayanana, Chem. Soc. Rev., 2008, 37, 1001; (b) H. N. Daghestani and B. W. Day, Sensors, 2010, 10, 9630.

2 (a) Y. Zhao, X. Zhao and Z. Gu, Adv. Funct. Mater., 2010, 20, 2970; (b) Y. Song, W. Wei and X. Qu, Adv. Mater., 2011, 23, 4215.

3 (a) J. H. Holtz and S. A. Asher, Nature, 1997, 389, 829; (b) K. Lee and S. A. Asher, J. Am. Chem. Soc., 2000, 122, 9534; (c) M. BenMoshe, V. L. Alexeev and S. A. Asher, Anal. Chem., 2006, 78, 5149; (d) X. Xu, A. V. Goponenko and S. A. Asher, J. Am. Chem. Soc., 2008, 130, 3113; (e) J. Zhang, L. Wang, J. Luo, A. Tikhonov, N. Kornienko and S. A. Asher, J. Am. Chem. Soc., 2011, 133, 9152.

4 (a) Y. Leeand and P. V. Braun, Adv. Mater., 2003, 15, 563; (b) D. Nakayama, Y. Takeoka, M. Watanabe and K. Kataoka, Angew. Chem., Int. Ed., 2003, 42, 4197; (c) K. Ueno, K. Matsubara, M. Watanabe and Y. Takeoka, Adv. Mater., 2007, 19, 2807; (d) E. Tian, J. Wang, Y. Zheng, Y. Song, L. Jiang and D. Zhu, J. Mater. Chem., 2008, 18, 1116; (e) N. Griffete, H. Frederich, A. Maître, M. M. Chehimi, S. Ravaine and C. Mangeney, J. Mater. Chem., 2011, 21, 13052; (f) Z. Wu, C. Tao, C. Lin, D. Shen and G. Li, Chem.-Eur. J., 2008, 14, 11358; $(g)$ N. Griffete, H. Frederich, A. Maître, S. Ravaine, M. M. Chehimi and C. Mangeney, Langmuir, 2012, 28, 1005; (h) W. Hong, X. Hu, B. Zhao, F. Zhang and D. Zhang, Adv. Mater., 2010, 22, 5043.

5 Y. J. Zhao, X. W. Zhao, J. Hu, J. Li, W. Y. Xu and Z. Z. Gu, Angew. Chem., Int. Ed., 2009, 48, 7350.

6 (a) B. V. Lotsch and G. A. Ozin, Adv. Mater., 2008, 20, 4097; (b) L. D. Boniacio, D. P. Puzzo, S. Breslav, B. M. Willey, A. McGeer and G. A. Ozin, Adv. Mater., 2009, 22, 1351; (c) M. Karaman, S. E. Kooi and K. K. Gleason, Chem. Mater., 2008, 20, 2262; (d) Z. Wang, J. Zhang, J. Xie, C. Li, Y. Li, S. Liang, Z. Tian, T. Wang, H. Zhang, H. Li, W. Xu and B. Yang, Adv. Funct. Mater., 2010, $20,3784$.

7 V. S.-Y. Lin, K. Motesharei, K.-P. S. Dancil, M. J. Sailor and M. R. Ghadiri, Science, 1997, 278, 840.

8 Y. Akahane, T. Asano, B. Song and S. Noda, Nature, 2003, 425, 944.

9 E. Chow, A. Grot, L. M. Mirkarimi, M. Sigalas and G. Girolami, Opt. Lett., 2004, 29, 1093.

10 (a) W. Huang, W. Qian and M. A. El-Sayed, Adv. Mater., 2008, 20, 733; (b) M. López-García, J. F. Galisteo-López, Á. Blancom, C. López and A. García-Martín, Adv. Funct. Mater., 2010, 20, 4338; (c) Y. Gao, A. D. Li, Z. B. Gu, Q. J. Wang, Y. Zhang, D. Wu, Y. F. Chen, N. B. Ming, S. X. Ouyang and T. Yu, Appl. Phys. Lett., 2007, 91, 031910; (d) C. Li, G. Hong and L. Qi, Chem. Mater., 2010, 22, 476; (e) G. Duan, W. Cai, Y. Luo and F. Sun, Adv. Funct. Mater., 2007, 17, 644.

11 (a) I. Tokarev, M. Orlov and S. Minko, Adv. Mater., 2006, 18, 2458; (b) S. Singamaneni, M. E. McConney and V. V. Tsukruk, Adv. Mater., 2010, 22, 1263; (c) I. Tokarev, M. Orlov, E. Katz and S. Minko, J. Phys. Chem. B, 2007, 111, 12141; (d) R. C. Hayward, B. F. Chmelka and E. J. Kramer, Macromolecules, 2005, 38, 7768-7783. 